\title{
SUPERCRITICAL STABILITY OF AN AXIALLY MOVING BEAM PART II: VIBRATION AND STABILITY ANALYSES
}

\author{
S.-J. Hwang and N. C. Perkins \\ Mechanical Engineering and Applied Mechanics, The University of Michigan, Ann Arbor, \\ Michigan 48109, U.S.A.
}

(Received 29 March 1990 and in final form 1 March 1991)

\begin{abstract}
This paper focuses on the stability of axially moving beam-like materials (e.g., belts, bands, paper and webs) which translate at speeds near to and above the so-called "critical speed stability limit." In the companion paper, a theoretical model for an axially moving beam was presented which accounted for geometrically non-linear beam deflections and the initial beam curvature generated by supporting wheels and pulleys. In that paper, analysis of steady response revealed that the beam possesses multiple, non-trivial equilibrium states when translating at supercritical speeds. The equations of motion are presently linearized about these equilibria and their stability is predicted from the eigenvalue problem for free response. Asymptotic and numerical solutions to the eigenvalue problem are presented for the respective cases of small and arbitrary equilibrium curvature. The solutions illustrate that the translating beam has multiple stable equilibrium states in the supercritical speed regime. The solutions confirm that the critical speed behavior for axially moving materials is extremely sensitive to system imperfections, such as initial curvature.
\end{abstract}

\section{INTRODUCTION}

Axially moving material problems $[1,2]$ consider the dynamic response of long, slender elements that are in a state of translation (e.g., translating bands, belts, cables, chains, fibers, paper, threads, webs, etc.). Traditional models of axially moving materials represent the translating element as either a taut string [3-5] or an Euler-Bernoulli beam [6-8] that is drawn perfectly straight under large tension. The analysis of such geometrically perfect systems leads to the prediction that the stability of axially moving materials is limited to translation speeds below a theoretical critical speed. At the critical speed, the translating element experiences a divergence-type instability.

Recent studies have demonstrated that critical speed behavior for flexible axially moving materials (e.g., cables, chains, fibers and threads) represents an idealized phenomenon that does not occur when imperfections are correctly accounted for in the model. For such systems, the initial sag of the element due to gravity creates a speed-tensioning effect that permits the sagged equilibrium to remain stable at very high translation speeds $[9,10]$. In addition, the initial sag creates the possibility of a second arch-like equilibrium which becomes stabilized at high translation speeds due to gyroscopic action [11, 12]. The studies focus on flexible elements and do not consider the flexural rigidity important in the modeling of translating beam-like elements (e.g., bands, belts, paper and webs). For these elements, imperfections in initial geometry naturally arise from the bending of the beam about its supporting pulleys or wheels. As demonstrated in Part I [13], the steady response of a translating beam depends strongly on the initial curvature induced by terminal 
bending. Bending leads to a single non-trivial beam equilibrium at subcritical speeds. At supercritical speeds, multiple non-trivial beam equilibria exist.

The purpose of this second part of the paper is to investigate the stability of small oscillations about these equilibria. The equations of motion are linearized about the beam equilibria, and local stability is predicted from the eigenvalue problem governing the free response. For the case of small equilibrium curvature, an asymptotic form of the eigenvalue problem is derived that is suitable for closed form analysis. The asymptotic solution accurately describes the stability of a translating beam equilibrium with small curvature, but fails to predict stability when the equilibrium has moderate to large curvature. A numerical stability analysis is presented for arbitrary equilibrium curvature and is based on a Galerkin discretization of the equations of motion.

\section{LINEARIZED EQUATIONS OF MOTION}

In Part I [13], a theoretical model was derived which describes the planar response of the translating beam shown in Figure 1. All quantities illustrated in this figure are nondimensionalized according to the variables defined in section 2 of Part I. The translating beam is subjected to steady tension $n$ and bending moments $m$ generated by supporting wheels or pulleys (not shown). In this model, the right support is stationary and the left support may move horizontally with partial restraint offered by finite support stiffness $k$. In response to the steady loading, the translating beam may undergo large static deformation leading to the equilibrium configuration $\chi^{i}$ shown in Figure 1. The beam particle translation velocity relative to $\chi^{i}$ is denoted by $c$.

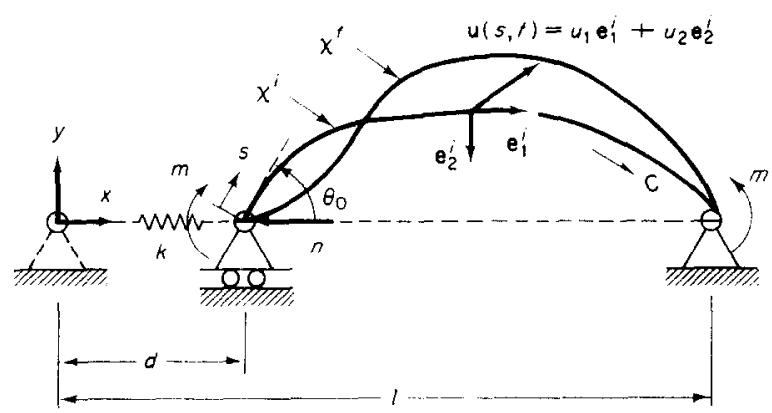

Figure 1. Definition diagram for a curved, axially moving beam. The beam centerline is shown in three states: (1) unbuckled configuration (dotted line); (2) buckled equilibrium configuration $\chi^{\prime}$; and (3) final configuration $\chi^{f}$. All variables are non-dimensionalized according to definitions given in section 2 of Part I [13].

In Part I, a detailed analysis of the non-linear deformation leading to $\chi^{i}$ was presented, and equilibrium solutions were derived for both subcritical and supercritical translation speeds. Here, attention is focused on small oscillations about equilibrium. The oscillations are described by the planar motion $\mathbf{u}(s, t)$ shown in Figure 1, where $s$ denotes the arc length co-ordinate measured along $\chi^{i}$ and $t$ denotes time. This motion $\mathbf{u}(s, t)=$ $u_{1} \mathbf{e}_{1}^{i}+u_{2} \mathbf{e}_{2}^{i}$ is resolved into components aligned with the local tangential, $\mathbf{e}_{1}^{i}$, and normal, $\mathbf{e}_{2}^{i}$, directions defined by the curve $\chi^{i}$. The non-linear equations of planar motion, Part $\mathbf{I}$, equations (15) and (16), were derived from Hamilton's principle, based on the assumptions that: (1) the beam is a homogeneous, one-dimensional elastic continuum obeying a linear stress-strain relationship; (2) extensions of the beam are described by the Lagrangian strain of the centerline; (3) the motion of the beam is restricted to the $X$ - $Y$ plane; (4) the beam may undergo large static deflections, and additional deflections from the curved 
equilibrium are described using a non-linear rod theory [14]; (5) rotary inertia due to bending and strain energy due to shear may be neglected assuming that the beam crosssectional dimensions are small compared to its length; (6) gravitational and dissipative forces may be neglected; and (7) the beam mass flux is constant.

To investigate the local stability of the equilibria, the non-linear equations of motion are linearized about an arbitrary equilibrium that is described by its curvature $\kappa(s)$ and tension $p(s)$. The linearized (non-dimensional) equations governing free, planar response are:

tangential component, $u_{1}$,

$$
\begin{aligned}
& {\left[\left(\gamma+p-c^{2}\right)\left(u_{1, s}-\kappa u_{2}\right)\right]_{, s}+\left[\kappa\left(c^{2}-p\right)\right]\left[u_{2, s}+\kappa u_{1}\right]+\kappa\left[u_{2, s}+\kappa u_{1}\right]_{\kappa s}} \\
& \quad=u_{1, r}+2 c\left[u_{1, s}-\kappa u_{2}\right]_{,}
\end{aligned}
$$

normal component, $u_{2}$,

$$
\begin{aligned}
& {\left[\kappa\left(\gamma+p-c^{2}\right)\right]\left[u_{1, s}-\kappa u_{2}\right]+\left[\left(p-c^{2}\right)\left(u_{2, s}+\kappa u_{1}\right)\right]_{, s}-\left[u_{2 . s}+\kappa u_{1}\right]_{. s s s}} \\
& \quad=u_{2, r t}+2 c\left[u_{2, s}+\kappa u_{1}\right]_{, t} .
\end{aligned}
$$

It should be noted that the linear equation of transverse motion for a perfectly straight translating beam [6-8] is obtained from equation (2) when the equilibrium is trivial; that is, when $\kappa(s)=0$ and $p(s)=n$. The linearized boundary conditions are extracted from the boundary conditions, Part 1 , equations (22)-(26), after incorporating the boundary conditions for equilibrium, Part I, equations (29) and (30). Assuming that the left support remains stationary after the beam attains its equilibrium, the linearized boundary conditions become:

$$
u_{1}=u_{2}=0, u_{2, s s}+\kappa u_{1 . s}=0, \quad \text { at } s=0,1 .
$$

Motion about a specific equilibrium is examined by prescribing the equilibrium curvature $\kappa(s)$ and tension $p(s)$ which appear as (non-constant) coefficients in equations (1) and (2). Exact expressions for $\kappa(s)$, in terms of elliptic integrals, are derived in Part 1 for both subcritical and supercritical translation speeds; see Part I, equations (43) - (48) and equations (56)-(59). The equilibrium tension follows from the solution for $\kappa(s)$ by evaluating equation (38) of Part I.

\section{ASYMPTOTIC ANALYSIS FOR SMALL CURVATURE}

The linearized equations of motion (1) and (2) are coupled, and have non-constant coefficients expressed in terms of elliptic integrals. As written, the complexity of these equations precludes their solution by analytical methods. However, an asymptotic form of the equations is amenable to closed form analysis and is valid for beams having small, but non-zero, equilibrium curvature. The asymptotic model is essentially that of a shallow translating arch, described in section 3.1 of Part I by the equilibrium solution

$$
\kappa(s)=\varepsilon\left[\left(1+\mathrm{e}^{-\mathrm{i} \alpha}\right) \mathrm{e}^{\mathrm{i} \alpha s}+\left(1+\mathrm{e}^{\mathrm{i} \alpha}\right) \mathrm{e}^{-\mathrm{i} \alpha s}\right], \quad 0 \leqslant s \leqslant 1 . \quad p(s)=n .
$$

The small parameter

$$
\varepsilon-\frac{m}{\left(1+\mathrm{e}^{-\mathrm{i} \alpha}\right)\left(1+\mathrm{e}^{\mathrm{i} \alpha}\right)} .
$$

is introduced to represent the order of magnitude of the equilibrium curvature and $\alpha=$ $\sqrt{c^{2}-n}$ is a measure of the beam translation speed. 
For low order flexural modes, the beam stretches in a quasi-static manner and the inertia terms in equation (1) may be neglected. This follows from the fact that the parameter $\gamma$ is extremely large and, therefore, the speed of propagation of longitudinal waves is substantially greater than those of low order flexural waves. $\dagger$ Using this spectral separation assumption in equation (1) and letting $\gamma$ be at least as large as $1 / \varepsilon$ leads to

$$
\left[u_{1,5}-\kappa u_{2}\right]_{, s}=0,
$$

where terms of first and higher order in $\varepsilon$ are neglected. Integration of equation (7) using $u_{1}(0, t)=u_{1}(1, t)=0$, yields

$$
u_{1}(s, t)=\int_{0}^{s} \kappa u_{2}(\xi, t) \mathrm{d} \xi-s \int_{0}^{t} \kappa u_{2}(\xi, t) \mathrm{d} \xi,
$$

which shows that the longitudinal motion is of order $\varepsilon$. Substituting equation (8) into equation (2) and neglecting terms of second and higher order in $\varepsilon$ provides the approximate equation for transverse motion:

$$
-u_{2, s s s s}-\alpha^{2} u_{2, s s}-\kappa(s) \gamma \int_{0}^{1} \kappa(\xi) u_{2}(\xi, t) \mathrm{d} \xi=u_{2, t t}+2 c u_{2, s t}
$$

The integral term in equation (9) accounts for the quasi-static stretching of the beam centerline and vanishes with vanishing initial curvature. To first order, the boundary conditions (3) are

$$
u_{2}=u_{2, s s}=0 \quad \text { at } s=0,1 .
$$

Using $u_{2}(s, t)=u(s) \mathrm{e}^{\mathrm{i} \omega t}$ in equations (9) and (10) results in the following eigenvalue problem governing the free, transverse response:

$$
\begin{gathered}
u^{\prime \prime \prime \prime}+\alpha^{2} u^{\prime \prime}+\mathrm{i} 2 c \omega u^{\prime}-\omega^{2} u=\kappa(s) \zeta, \quad 0<s<1, \\
u(0)-u(1)-0, \quad u^{\prime \prime}(0)=u^{\prime \prime}(1)=0,
\end{gathered}
$$

where

$$
\zeta=-\gamma \int_{0}^{1} \kappa(\xi) u(\xi) \mathrm{d} \xi .
$$

Exact analysis of equations (11)-(13) provides the eigenvalues $\omega$ which govern the stability of small transverse oscillations. For the case $\zeta \neq 0, \ddagger$ the general solution to equation (11) is

$$
\frac{u(s)}{\zeta}=C_{1} \mathrm{e}^{\lambda_{1} s}+C_{2} \mathrm{e}^{\lambda_{2} s}+C_{3} \mathrm{e}^{\lambda_{3} s}+C_{4} \mathrm{e}^{\lambda_{4} s}+F \mathrm{e}^{\mathrm{i} \theta s}+G \mathrm{e}^{-\mathrm{i} \alpha s},
$$

provided that the complex exponents $\lambda_{1}, \lambda_{2}, \lambda_{3}$ and $\lambda_{4}$ satisfy

$$
\lambda^{4}+\alpha^{2} \lambda^{2}+\mathrm{i} 2 c \omega \lambda-\omega^{2}=0
$$

† From section 2 of Part $1, \gamma=A L^{2} / I$, where $A, L$ and $I$ are the beam cross-sectional area, overall length and area moment of inertia about the out-of-plane axis, respectively.

$\ddagger$ For the case $\zeta=0$, the eigenvalue problem of equations (11)-(13) reduces to that for a straight beam and the solution follows references [6-8]. 
In equation (14),

$$
F=\frac{-m}{\left(1+\mathrm{e}^{\mathrm{i} \alpha}\right)\left(2 c \alpha \omega+\omega^{2}\right)}, \quad G=\frac{m}{\left(1+\mathrm{e}^{-\mathrm{i} \alpha}\right)\left(2 c \alpha \omega-\omega^{2}\right)},
$$

and $C_{1}, C_{2}, C_{3}, C_{4}$ and $\zeta$ are (undetermined) constants. Substituting the solution (14) into the boundary conditions (12) leads to the following set of linear equations for solution of $C_{1}, C_{2}, C_{3}$ and $C_{4}$ :

$$
\left[\begin{array}{cccc}
1 & 1 & 1 & 1 \\
\lambda_{1}^{2} & \lambda_{2}^{2} & \lambda_{3}^{2} & \lambda_{4}^{2} \\
\mathrm{e}^{\lambda_{1}} & \mathrm{e}^{\lambda_{2}} & \mathrm{e}^{\lambda_{3}} & \mathrm{e}^{\lambda_{4}} \\
\lambda_{1}^{2} \mathrm{e}^{\lambda_{1}} & \lambda_{2}^{2} \mathrm{e}^{\lambda_{2}} & \lambda_{3}^{2} \mathrm{e}^{\lambda_{3}} & \lambda_{4}^{2} \mathrm{e}^{\lambda_{4}}
\end{array}\right]\left(\begin{array}{c}
C_{1} \\
C_{2} \\
C_{3} \\
C_{4}
\end{array}\right)=\left[\begin{array}{c}
-(F+G) \\
\alpha^{2}(F+G) \\
-\left(F \mathrm{e}^{\mathrm{i} \alpha}+G \mathrm{e}^{-i \alpha}\right) \\
\alpha^{2}\left(F \mathrm{e}^{\mathrm{i} \alpha}+G \mathrm{e}^{-i \alpha}\right)
\end{array}\right]
$$

Final evaluation of equation (13) leads to the characteristic equation for the solution of the eigenvalues $\omega_{j}, j=1,2, \ldots$,

$$
\begin{aligned}
1-\left(\alpha^{2}-\gamma\right)\left\{\frac{m}{1+\mathrm{e}^{\mathrm{i} \alpha}}\left[\sum_{l=1}^{4} \frac{C_{l}}{\lambda_{l}+\mathrm{i} \alpha}\left(\mathrm{e}^{\lambda_{1}+\mathrm{i} \alpha}-1\right)+G+\frac{F}{2 \mathrm{i} \alpha}\left(\mathrm{e}^{2 \mathrm{i} \alpha}-1\right)\right]\right. \\
\left.+\frac{m}{1+\mathrm{e}^{-\mathrm{i} \alpha}}\left[\sum_{l=1}^{4} \frac{C_{l}}{\lambda_{l}-\mathrm{i} \alpha}\left(\mathrm{e}^{\lambda_{l}-\mathrm{i} \alpha}-1\right)+F+\frac{G}{-2 \mathrm{i} \alpha}\left(\mathrm{e}^{-2 \mathrm{i} \alpha}-1\right)\right]\right\}=0 .
\end{aligned}
$$

The (generally complex) eigenvalues are determined numerically by finding the roots of equation (18) using the standard Newton-Raphson iteration. For this purpose, the characteristic equation is evaluated as follows. For a given value of $\omega$, the complex exponents $\lambda_{1}-\lambda_{4}$ are determined numerically as the roots of equation (15). The constants $C_{1}-C_{4}$ are then found from the linear equation (17) and are used to evaluate the characteristic equation (18).

An example demonstrates that these eigenvalues are extremely sensitive to equilibrium curvature when the beam is translating near the fundamental critical speed of a simply supported straight beam which is given by $c=\sqrt{\pi^{2}+n}[6,7]$. In Figure 2(a), the first two natural frequencies (real $\omega_{1}$ and $\omega_{2}$ ) are plotted versus beam translation speed for the case of a slight bending moment $m=0.25$ and a large tension $n=100$. For comparison, the first two frequencies of vibration about the trivial equilibrium are shown by the dotted curves $[6,7]$. For the small value of $m$ considered here, the frequencies of the "straight beam" and the "shallow arch" are nearly identical for translation speeds below and away from the fundamental critical speed. Near the fundamental critical speed, however, the solutions diverge in a significant manner. While the natural frequencies of the straight beam continue to decrease, those of the shallow arch suddenly increase in the neighborhood of the fundamental critical speed. This sudden stiffening derives from the rapid increase in equilibrium curvature near the fundamental critical speed. This effect is illustrated in Figure 2(b), where $|\varepsilon|$ is also plotted as a function of translation speed.

It was shown in Part I that multiple beam equilibrium states exist above the fundamental critical speed for the straight beam : see Figure 3, which shows how the angle of inclination of the equilibrium curve at the left support depends on translation speed. In the supercritical speed region of Figure 3, the linear equilibrium solution (4) remains a good approximation only to that equilibrium which is nearest the trivial solution. The nearness of the linear solution to the near-trivial equilibrium is reflected in Figure 2(a) where, above the fundamental critical speed for the straight beam, the freqencies of the shallow arch decrease in a manner similar to those of the straight beam. However, note that the fundamental frequency for the shallow arch vanishes at a translation speed near the second 


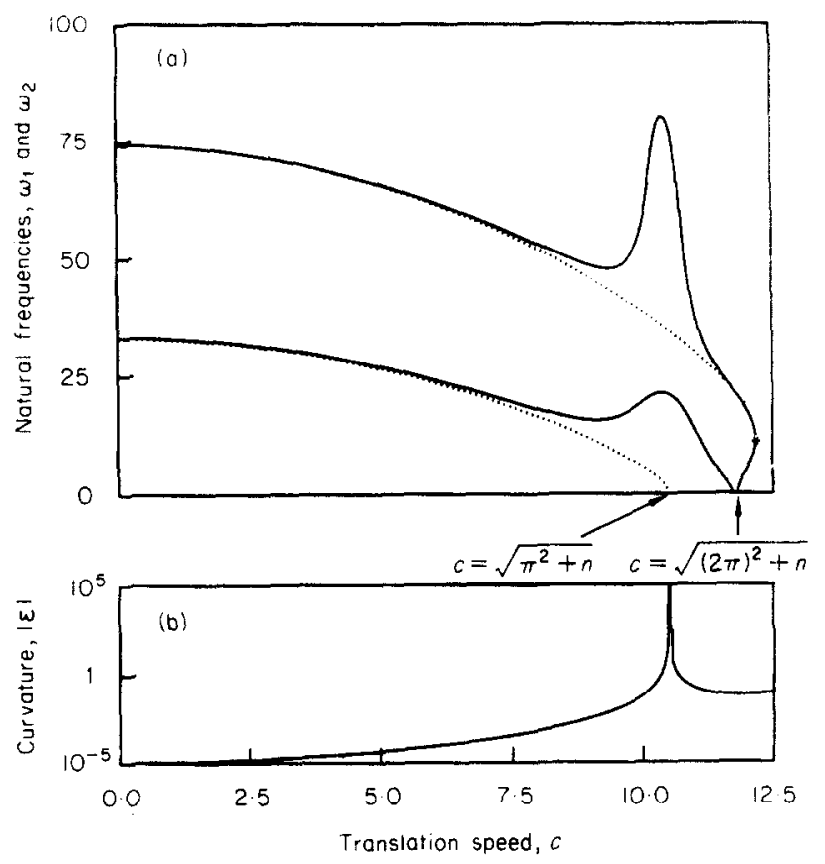

Figure 2. The first two natural frequencies for a translating beam with small curvature ( - - $)$ and no curvature $(\cdot \cdots)$ are shown in (a). In this example, $m=0 \cdot 25$ and $n=100$. The magnitude of curvature $|\varepsilon|$ is shown in (b).

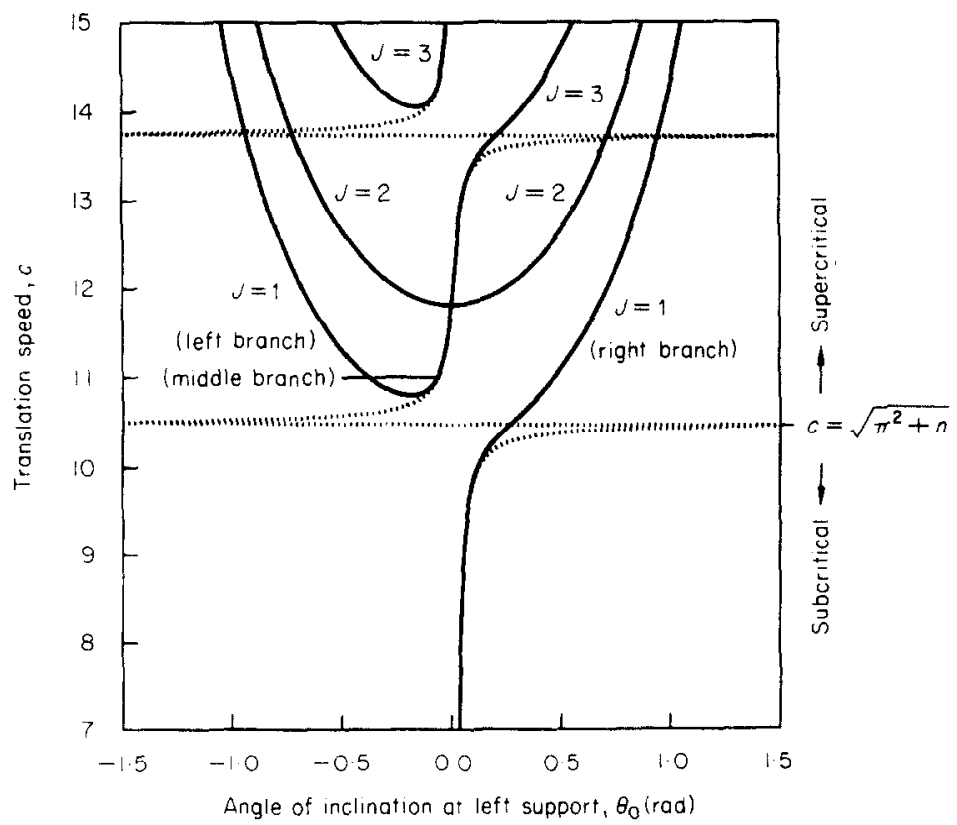

Figure 3. Lincar ( . . . ) and non-linear ( $\ldots-$ ) equilibrium solutions shown in the subcritical and supercritical speed regions for the case $m=0 \cdot 25, n=100$ and $k=0$. For the non-linear solution, $J$ denotes the solution order. Refer to Part I for a discussion of the folded and unfolded bifurcations. 
critical speed for the straight beam. Thus, the range of stable operating speeds for the shallow arch substantially exceeds that of the straight beam. The second frequency for the shallow arch follows closely that of the straight beam after the fundamental critical speed for the straight beam. In particular, the second critical speed, $c=\sqrt{(2 \pi)^{2}+n}$, is identical for the two systems and the first two eigenvalues become complex (flutter instability occurs) beyond the point marked by the asterisk in the figure.

The sensitivity of the first two natural frequencies of the shallow arch to changes in the bending moment is illustrated in Figure 4. Increasing the bending moment from $m=0$ to

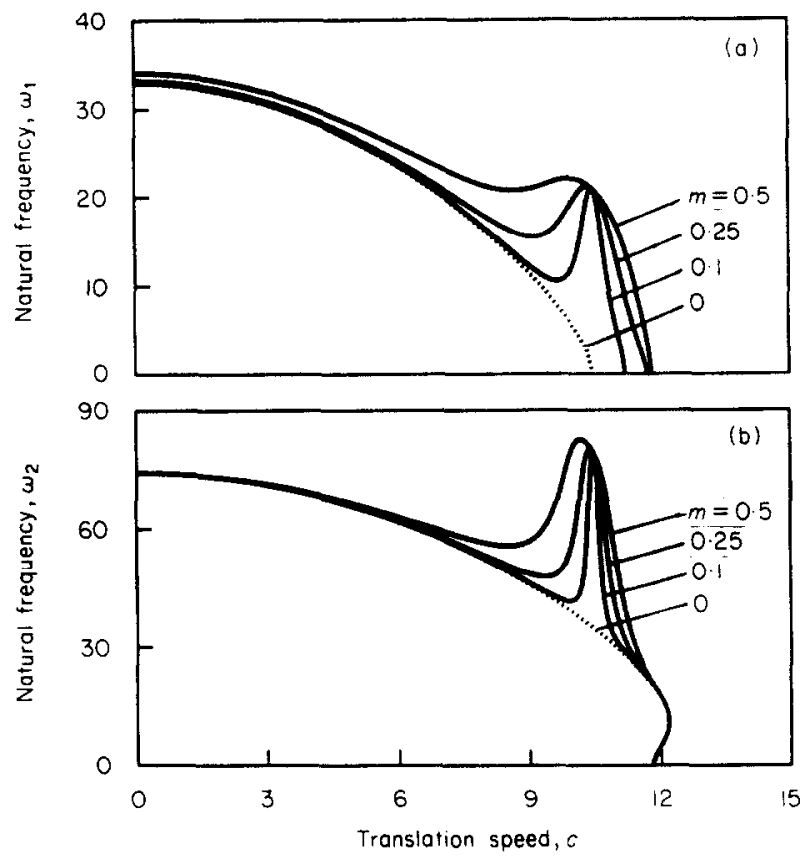

Figure 4. Sensitivity of the first two natural frequencies to changes in bending moment. In all cases, $n=100$ was used. - Shallow arch; $\cdots \cdots$, straight beam.

$m=0.5$ leads to a substantial increase in the fundamental frequency, as shown in Figure 4(a). The increase becomes very pronounced near the fundamental critical speed for the straight beam and produces a noticeable increase in the fundamental critical speed for the shallow arch. Similar increases in the second natural frequency are observed in Figure 4(b) near the fundamental critical speed for the straight beam. For the second natural frequency, however, no increases are observed at low subcritical speeds or in a neighborhood of the second critical speed. Since the second mode of vibration is antisymmetric, the (symmetrically) applied bending moments have no influence on buckling in this mode; also refer to Figure 3 and to the discussion in Part $I$ regarding the folded and unfolded bifurcations of the equilibrium solutions.

Overall, it is illustrated in Figures 2 and 4 that the translating beam frequency spectrum is extremely sensitive to equilibrium curvature, particularly when the beam is translating at near-critical speeds. The asymptotic solution predicts that equilibrium curvature has a stabilizing effect which leads to large increases in the natural frequencies near the fundamental critical speed for the traditional, straight beam model. While the asymptotic solution qualitatively captures this important trend, it cannot be expected to remain accurate when the equilibrium curvature becomes large. In Figure $2(\mathrm{~b})$ it is shown that $|\varepsilon|$ becomes unbounded at the fundamental critical speed and the asymptotic solution is certainly 
suspect. Furthermore, since the asymptotic model describes small oscillations about a single and near-trivial equilibrium, it cannot predict the stability of the multiple, nontrivial equilibria known to exist above the fundamental critical speed; refer to the left-and right-branch equilibria labeled in Figure 3.

\section{NUMERICAL ANALYSIS FOR ARBITRARY CURVATURE}

A numerical method is adopted to determine the stability of a translating beam with arbitrary equilibrium curvature. Galerkin's method is used to discretize the linear equations of motion and stability is determined from the eigensolutions of the discrete model.

Consider the $R$-term admissible series representations for $u_{1}$ and $u_{2}$ of the form:

$$
u_{i}(s, t)=\sum_{j=1}^{R} \phi_{i j}(t) \Theta_{j}(s), \quad i=1,2
$$

where $\Theta_{j}(s)=\sqrt{2} \sin (j \pi s)$. Substitution of equation (19) into equations (1) and (2), and application of the Galerkin method, provides a set of $2 R$ coupled, ordinary differential equations for solving for the generalized co-ordinates $\phi_{i j}(t)$ :

$$
\mathbf{M} \ddot{\vec{\phi}}+\mathbf{G} \dot{\vec{\phi}}+\mathbf{K} \vec{\phi}=\overrightarrow{0} \text {. }
$$

The elements of the matrices $\mathbf{M}, \mathbf{G}$ and $\mathbf{K}$, which are given in the Appendix, are evaluated by numerical quadrature. These matrices depend on the equilibrium state under consideration and are evaluated using the elliptic integral solutions for $k(s)$ and $p(s)$ given in Part I. Using $\vec{\phi}(t)=\vec{Q} \mathrm{e}^{\omega t}$ in equation (20) leads to the discrete eigenvalue problem governing free response:

$$
\left[\omega^{2} \mathbf{M}+\omega \mathbf{G}+\mathbf{K}\right] \vec{Q}=\overrightarrow{0} .
$$

The natural frequencies and mode shapes for the translating beam are obtained numerically from equation (21). In this discrete formulation, the natural frequencies of the translating beam are given by the imaginary parts of the eigenvalues, $\operatorname{Im}\left[\omega_{l}\right], l=1,2, \ldots, 2 R$, and the mode shapes are obtained from the eigenvectors $\vec{Q}_{l}, l=1,2, \ldots, 2 R$, through equation (19). A divergence instability for mode $l$ is identified when $\operatorname{Im}\left(\omega_{l}\right) \rightarrow 0$. In calculations, the series size $R=10$ was and the eigensolutions described below have fully converged.

As a primary example, consider the stability of a translating beam subjected to the slight bending moment $m=0.25$, as illustrated by the equilibrium solutions of Figure 3. In Figure 5 are shown the fundamental natural frequencies of vibration as functions of translation speed in both the subcritical and supercritical speed regions. In the supercritical speed region, three curves are shown which depict separately the fundamental frequencies of vibration about the right-branch, middle-branch and left-branch equilibria shown in Figure 3 for the (fundamental) equilibrium solution $J=1$. The fundamental frequency of vibration about the trivial equilibrium is also shown to facilitate a comparison with the traditional model of a perfectly straight beam $[6,7]$. The solid curve in Figure 5, which depicts the fundamental frequency of vibration about the right-branch equilibrium, clearly shows that this equilibrium nover loses stability over the indicated speed range. While the fundamental frequency decreases in the subcritical speed region, it rapidly increases in the neighborhood of the fundamental critical speed for the trivial equilibrium. Again, this effect derives from the stiffening of the beam as its curvature markedly increases near this fundamental critical speed; see region of rapid growth of $\theta_{0}$ for the right-branch equilibrium $(J=1)$ of Figure 3. Likewise, the left-branch equilibrium remains stable after it has formed in the supercritical speed region, as seen by the dashed curved of Figure 5. The middle-branch equili- 


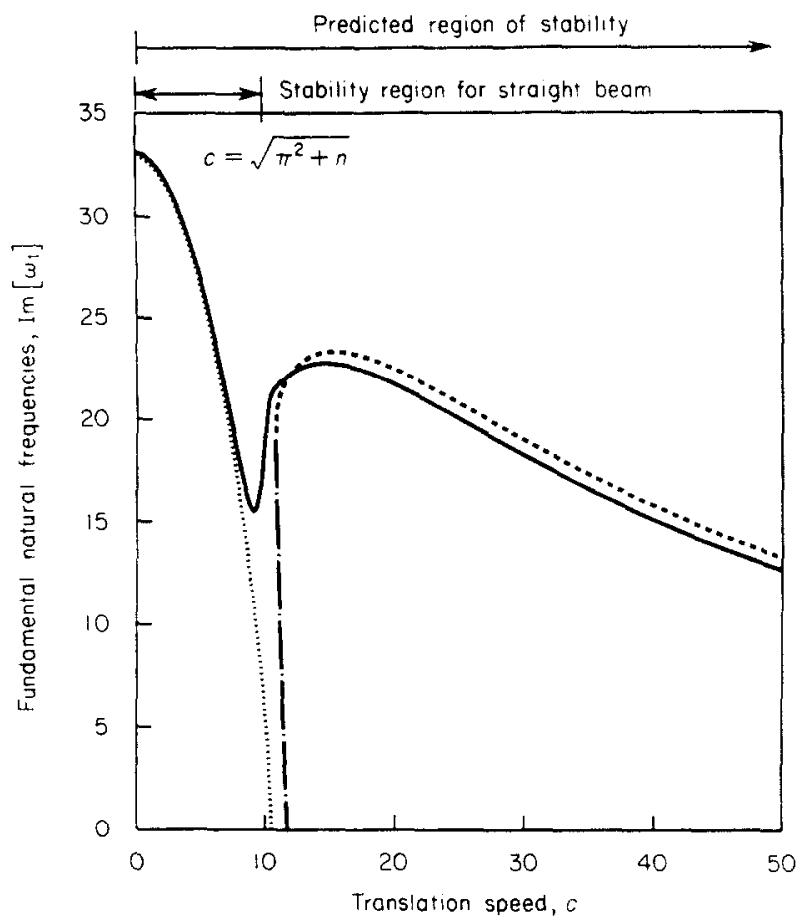

Figure 5. Stability of a curved, axially moving beam. The fundamental frequencies of vibration are plotted versus translation speed for vibration about the right- (_- ), middle- (- - - ) and left-branch (- - ) equilibria $(J=1)$ of Figure 3. In this example, $m=0.25, n=100$ and $k=0$. The dotted curve $(\cdots)$ represents the fundamental frequency of vibration about trivial equilibrium.

brium, however, docs experience a divergence instability, but at a translation speed above the fundamental critical speed for the trivial equilibrium, as shown by the dash-dot curve of Figure 5.

In Figure 5, the fundamental frequencies for the two stable equilibria (right and leftbranch) are nearly identical for large supercritical speeds. This results from the fact that their respective equilibrium shapes become nearly mirror images at large translation speeds; observe the magnitude of $\theta_{0}$ for the right- and left-branch equilibria in Figure 3. In Figure 5, it can be noted that the fundamental frequency associated with the trivial equilibrium appears to provide a lower bound to that associated with the right-branch equilibrium for subcritical speeds. This behavior can again be anticipated from Figure 3, which shows that the trivial equilibrium itself is a reasonable approximation to the rightbranch equilibrium in the subcritical speed region. Upon comparing Figures 2(a) and 5 , the fundamental frequency predicted earlier by the asymptotic analysis follows that for the right-branch equilibrium below and just above the fundamental critical speed for the trivial equilibrium, and then follows that for the middle-branch equilibrium for higher translation speeds. Thus, as expected, the asymptotic solution describes the linear response about the equilibrium state which is nearest to the trivial equilibrium. Finally, it should be noted that the fundamental eigenvalues computed for the higher-order equilibrium solutions depicted in Figure 3 for $J=2,3, \ldots$ indicate that they are all unstable. For a similar class of stationary beams, Love [14] demonstrates that the stable fundamental equilibrium minimizes the strain energy and has the fewest number of inflection points.

The natural frequencies of the second and higher order vibration modes follow trends similar to that of the fundamental mode as illustrated by the results in Figure 6 . The first 


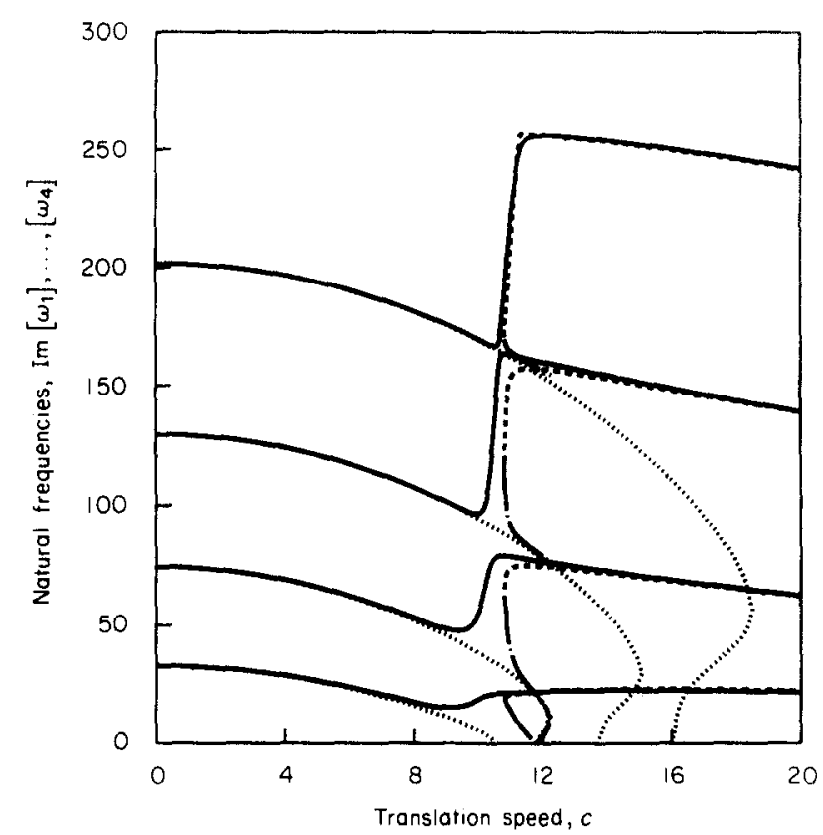

Figure 6. Higher order natural frequencies. The plot shows the first four natural frequencies of vibration about the right- (- - , middle- $(-\cdot-)$ and left-branch $(--)$ equilibria for $J=1$ and a portion of right-branch equilibrium (- - ) for $J=3$ of Figure 3. In this example, $m=0 \cdot 25, n=100$ and $k=0$. Dotted curves ( . . .) represent natural frequencies for vibration about trivial equilibrium.

four natural frequencies for vibration about the right-, middle-, and left-branch equilibria are plotted versus translation speed together with those associated with the trivial equilibrium $[6,7]$. An important point to note from this plot is that the fundamental frequency associated with the middle-branch equilibrium vanishes precisely at the second critical speed associated with the trivial equilibrium. Thus, it is the second critical speed for the trivial equilibrium which ultimately governs the stability of the middle-branch equilibrium. It was seen from Figure 3 that the bifurcation of the non-linear equilibrium solution occurring at the second critical speed is unaffected by the (symmetrically applied) bending moments and remains folded. Indeed, at the second critical speed, the middle-branch equilibrium is the trivial equilibrium. At this speed, the middle-branch equilibrium solution bifurcates (see Figure 3 ) and the "near-trivial" equilibrium thereafter is described by the right branch for $J=3$. For speeds just above the second critical speed, this equilibrium remains nearly trivial, and the natural frequencies shown in Figure 6 reproduce those for the trivial equilibrium in this region which includes the flutter instability. For clarity, in Figure 6, the present eigensolution calculation for this unstable equilibrium was terminated for higher translation speeds.

The stable fundamental modes for vibration about the right-, middle- and left-branch equilibria are illustrated in Figure 7 for the case in which the translation speed is $10 \%$ greater than the fundamental critical speed for the trivial equilibrium. The dotted curves depict the supercritical equilibrium shapes and the solid curves depict the beam at various times as it oscillates at its natural frequency over one period. As with all axially moving material (gyroscopic) systems, the free response has non-uniform phase and is described by complex eigenfunctions [1]. Also note that, at this somewhat modest supercritical translation speed, the right- and left-branch equilibria are already nearly mirror images.

The influence of the support stiffness on the natural frequency spectrum is illustrated in Figure 8 , which shows the first four natural frequencies for the case of a large support 


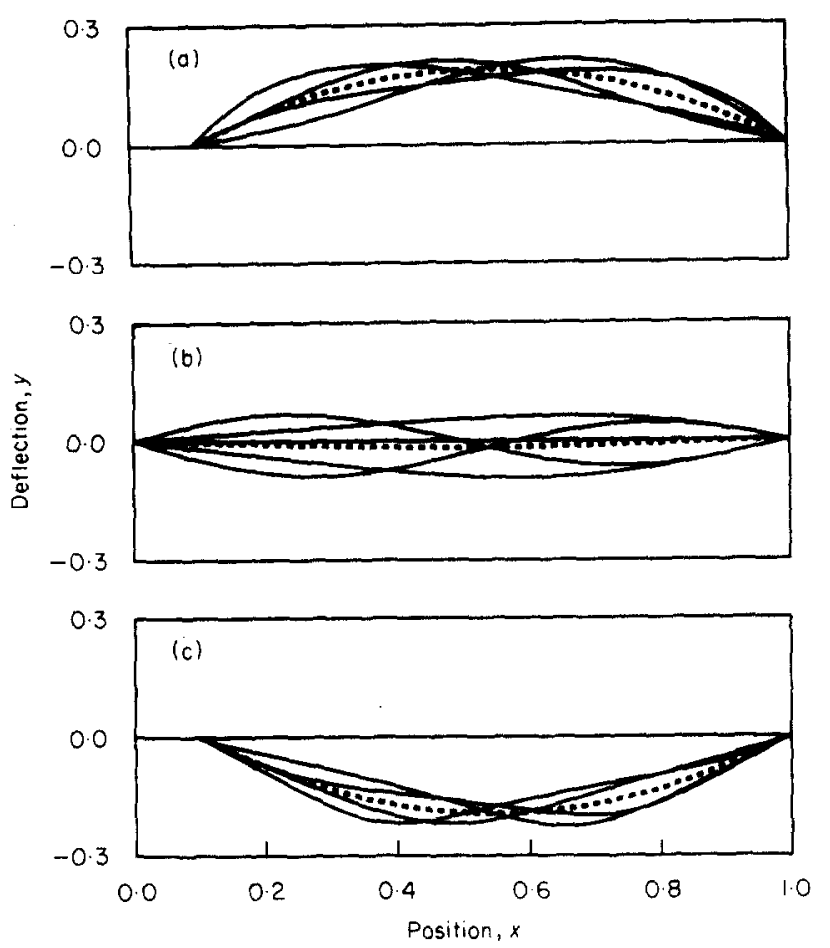

Figure 7. Fundamental mode shapes at supercritical speed $c / \sqrt{\pi^{2}+n}=1 \cdot 10$. Results are shown for vibration about (a) right-, (b) middle- and (c) left-branch equilibria $(J=1)$ in Figure 3. Solid curves depict the beam profile at times $t=0,0.25 \tau, 0.75 \tau$ and $\tau$, where $\tau$ is the fundamental period of oscillation. Dashed curves depict the equilibrium beam profiles. In all cases $m=0 \cdot 25, n=100$ and $k=0$.

stiffness $k=20000$ with $m=0.25$ and $n=100$. Again, frequencies of vibration about the trivial, right-, middle- and left-branch equilibria are shown. By comparison with Figure 6 $(k=0)$, it is observed that large support stiffness leads to more gradual increases in the frequencies near the fundamental critical speed for the trivial equilibrium. This behavior is anticipated from Figure 5 of Part I, which shows that the increases in $\theta_{0}$ for the right-, middle- and left-branch equilibria are much less abrupt for large $k$. As also noted there, large support stiffness retards the formation of the left- and middle-branch equilibria (there is a $8 \%$ increase of speed where the middle- and left-branch frequency loci met in Figure 8 ) and leads to a slight increase in the second bifurcation speed for the non-linear equilibrium solution ( $2 \%$ increase of the critical speed for the middle-branch equilibrium in Figure 8 ).

\section{SUMMARY AND CONCLUSIONS}

Linearized equations of motion for an axially moving beam are presented which account for the equilibrium beam curvature generated by supporting wheels and pulleys. The equations follow from the model derived in [13] which considers the geometrically nonlinear beam deflections expected at critical and supercritical translation speeds. Analysis of the equations of equilibrium [13] reveals that the beam undergoes large static deflections near the fundamental critical speed predicted by the traditional model of a perfectly straight translating beam [6-8]. Slightly above this speed, three fundamental equilibrium states exist, which are referred to herein as the right-branch, middle-branch and left-branch equilibria. These results confirm that critical speed behavior for axially moving materials is extremely sensitive to system imperfections, such as initial curvature. 


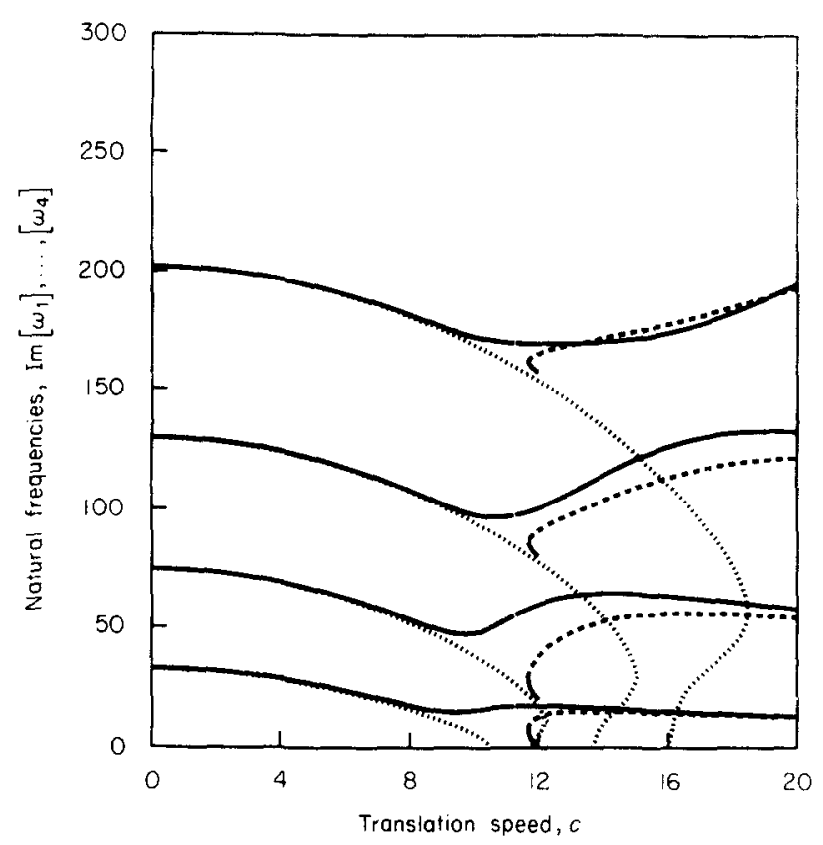

Figure 8. The effect of support stiffness on the frequency spectrum for the case of $m=0 \cdot 25, n=100$ and $k=$ 20000 . The plot shows first four natural frequencies of vibration about the right- $(-\infty-)$, middle- $\left(-\cdot-{ }_{-}\right)$and left-branch $(--)$ equilibria $(I=1)$. Dotted curves $(\cdots)$ ) represent natural frequencies for vibration about trivial equilibrium.

The equations of motion are linearized about the equilibria, and their stability is determined from (1) an asymptotic analysis for small equilibrium curvature, and (2) a numerical analysis for arbitrary equilibrium curvature. For the idealized case of no initial curvature, the trivial equilibrium experiences a divergence instability at the fundamental critical speed $[6,7]$. The present study shows that the beam then buckles and may form either the right or left-branch equilibria which are both stable in the supercritical speed region. When the beam possesses any degree of initial curvature, however, no divergence instability exists at the traditional fundamental "critical" speed. In such cases, the equilibrium state in the subcritical speed region is a continuous extension of the right-branch equilibrium, and the beam never loses stability. The left-branch equilibrium also remains stable after it appears in the supercritical speed region. In such instances, the initial conditions ultimately determine whether the beam oscillates about the left-branch equilibrium, the right-branch equilibrium or about both equilibria. Determination of the associated domains of attraction in the space of initial conditions could be achieved through analysis of the non-linear equations of motion.

\section{ACKNOWLEDGMENTS}

The authors gratefully acknowledge the support of the University of Michigan Office of the Vice President for Research, and the partial support of the U.S. Office of Naval Research Young Investigator Program.

\section{REFERENCES}

1. C. D. Mote, JR. 1972 Shock and Vibration Digest 4, 2-11. Dynamic stability of axially moving materials. 
2. J. A. Wickert and C. D. Mote, JR. 1988 Shock and Vibration Digest 20, 3-13. Current research on the vibration and stability of axially-moving materials.

3. R. A. SACK 1954 British Journal of Applied Physics 5, 224-226. Transverse oscillations in travelling strings.

4. S. Mahalingam 1957 British Journal of Applied Physics 8, 145-148. Transverse vibrations of power transmission chains.

5. W. L. Miranker 1960 IBM Journal of Research and Development 4, 36-42. The wave equation in a medium in motion.

6. T. Chubachi 1957 Bulletin of the Japan Society of Mechanical Engineers 23, 205-210. Lateral vibration of axially moving wire or belt form materials.

7. C. D. Mote, JR. 1965 Journal of the Franklin Institute 279, 430-444. A study of handsaw vibrations.

8. A. SiMPSON 1973 Journal of Mechanical Engineering Science 15, 159-164. Transverse modes and frequencies of beams translating between fixed end supports.

9. A. Simpson 1972 Journal of Sound and Vibration 20, 177-189. On the oscillatory motions of translating elastic cables.

10. N. C. Perkins and C. D. Mote, JR. 1987 Journal of Sound and Vibration 114, 325-340. Threedimensional vibration of travelling elastic cables.

11. N. C. Perkins and C. D. Mote, JR. 1989 Journal of Sound and Vibration 128, 397-410. Theoretical and experimental stability of two translating cable equilibria.

12. N. C. Perkins 1989 Journal of Sound and Vibration 135, 375-383. Asymptotic analysis of a translating cable arch.

13. S.-J. Hwang and N. C. Perkins 1992 Journal of Sound and Vibration 154, 381-396. Supercritical stability of an axially moving beam, part I: model and equilibrium analysis.

14. A. E. H. Love 1927 A Treatise on the Mathematical Theory of Elasticity. New York: Dover, 1944 republication of fourth edition. See Chapters 18 and 21.

\section{APPENDIX}

The discretized equations of motion (20) are given by

$$
\mathbf{M}\left[\begin{array}{c}
\ddot{\phi}_{11} \\
\ddot{\phi}_{12} \\
\vdots \\
\ddot{\phi}_{2 R}
\end{array}\right]+\mathbf{G}\left[\begin{array}{c}
\dot{\phi}_{11} \\
\dot{\phi}_{12} \\
\vdots \\
\dot{\phi}_{2 R}
\end{array}\right]+\mathbf{K}\left[\begin{array}{c}
\phi_{11} \\
\phi_{12} \\
\vdots \\
\phi_{2 R}
\end{array}\right]=\overrightarrow{0}
$$

where the $2 R \times 2 R$ coefficient matrices are

$$
\mathbf{M}=\left[\begin{array}{cc}
\mathbf{M}_{11} & 0 \\
0 & \mathbf{M}_{22}
\end{array}\right], \quad \mathbf{G}=\left[\begin{array}{ll}
\mathbf{G}_{11} & \mathbf{G}_{12} \\
\mathbf{G}_{21} & \mathbf{G}_{22}
\end{array}\right], \quad \mathbf{K}=\left[\begin{array}{ll}
\mathbf{K}_{11} & \mathbf{K}_{12} \\
\mathbf{K}_{21} & \mathbf{K}_{22}
\end{array}\right] .
$$

Letting $\langle a, b\rangle=\int_{0}^{l} a(s) b(s) \mathrm{d} s$, the $R \times R$ submatrices $\mathbf{M}_{11}, \ldots, \mathbf{K}_{22}$ are

$$
\begin{gathered}
\mathbf{M}_{11}(i, j)-\mathbf{M}_{22}(i, j)=\left\langle\Theta_{i}, \Theta_{j}\right\rangle=\delta_{i j} \quad \text { (Kronecker's delta), } \\
\mathbf{G}_{11}(i, j)=\mathbf{G}_{22}(i, j)=2 c\left\langle\Theta_{i}, \Theta_{j}^{\prime}\right\rangle, \\
\mathbf{G}_{12}(i, j)=-\mathbf{G}_{21}(j, i)=-2 c\left\langle\Theta_{i}, \kappa \Theta_{j}\right\rangle, \\
\mathbf{K}_{11}(i, j)=\left\langle\left(\kappa \Theta_{i}\right)^{\prime},\left(\kappa \Theta_{j}\right)^{\prime}\right\rangle+\left\langle\Theta_{i}, \kappa^{2}\left(p-c^{2}\right) \Theta_{j}\right\rangle+\left\langle\Theta_{i}^{\prime},\left(p+\gamma-c^{2}\right) \Theta_{j}^{\prime}\right\rangle . \\
\mathbf{K}_{22}(i, j)=\left\langle\Theta_{i}^{\prime}, \Theta_{j}^{\prime \prime}\right\rangle+\left\langle\Theta_{i}^{\prime},\left(p-c^{2}\right) \Theta_{j}^{\prime}\right\rangle+\left\langle\Theta_{i}, \kappa^{2}\left(p+\gamma-c^{2}\right) \Theta_{j}\right\rangle, \\
\mathbf{K}_{12}(i, j)=\mathbf{K}_{21}(j, i)=\left\langle\left(\kappa \Theta_{i}\right)^{\prime}, \Theta_{j}^{\prime \prime}\right\rangle+\left\langle\Theta_{i}^{\prime}, \kappa\left(p-c^{2}\right) \Theta_{j}\right\rangle+\left\langle\Theta_{i}^{\prime},-\kappa\left(p+\gamma-c^{2}\right) \Theta_{j}\right\rangle,
\end{gathered}
$$

where $i, j=1,2, \ldots, R$ and $\Theta_{j}=\sqrt{2} \sin (j \pi s)$. The equilibrium curvature $\kappa(s)$ and tension $p(s)$ are obtained from the solution of the equilibrium problem [13]. 